\section{Antidepressant use during first trimester not associated with risk of major congenital malformations}

\section{QUESTION}

Question: Does duration of antidepressant use during the first trimester of pregnancy increase the risk of major congenital malformations?

People: 2329 women, pregnant between 1 January 1998 and 31 December 2002, meeting the following inclusion criteria: one diagnosis of psychiatric disorder before pregnancy (ICD-9); antidepressant use for 30 days or more in the year prior to pregnancy; aged 15-45 years at the beginning of pregnancy; and live or still birth at the end of pregnancy. Women were identified using three administrative databases of the Province of Quebec (RAMO, MED-ECHO and ISO databases). Mailed questionnaires were used to elicit information on confounding variables such as income, education, ethnicity and lifestyle factors. Exclusion criteria: women with mental retardation, certain psychotic or non-psychotic conditions; abortion and miscarriage. Setting: Province of Quebec, Canada; recruitment January 1998-December 2002.

Risk factors: Exposure to antidepressants during the first trimester of pregnancy (0-14 weeks) was assessed for cases and controls. Women filling prescriptions for at least one antidepressant during the first trimester or before the first day of gestation where duration of treatment lasted into the first trimester were included in the exposure group. Exposure was further classified by duration of use (defined as the number of days exposed in the first trimester: 0 days, 1-30 days, 3160 days and $\geqslant 61$ days); therapeutic class and co-exposures. Analyses were adjusted for maternal age, welfare, urban dweller, living alone, comorbidities, hypertension and diabetes before and during pregnancy, gender of baby, prenatal visits and year of pregnancy.

Outcomes: Cases defined as any major congenital malformation diagnosed during the first year of the offspring's life. Controls defined as women with infants with no major congenital malformations.

\section{METHODS}

Design: Case control study.

Follow-up period: Retrospective assessment of 21 month period (pregnancy plus offspring's first year of life).

\section{MAIN RESULTS}

At least one major congenital malformation was identified in 189 (8\%) infants, with 265 malformations being reported in total. Using an antidepressant during the first trimester was not associated with a major congenital malformation (adjusted OR 1.10, 95\% CI 0.75 to 1.62). There was also no association with risk of congenital malformation in analyses of different durations of exposure to an antidepressant during the first trimester (1-30 days' exposure: adjusted OR 1.23, 95\% CI 0.77 to 1.98 ; 31-60 days' exposure: adjusted OR 1.03, $95 \%$ CI 0.63 to $1.69 ; \geqslant 61$ days' exposure: adjusted OR 0.92 , $95 \%$ CI 0.50 to 1.69$)$. No association was found between major congenital malformations and class of antidepressant used during the first trimester.

\section{CONCLUSIONS}

Offspring of women with psychiatric disorders taking antidepressants during the first trimester of pregnancy are not at increased risk of major congenital malformations.

\section{ABSTRACTED FROM}

Ramos E, St-André M, Rey E, et al. Duration of antidepressant use during pregnancy and risk of major congenital malformations. Br J Psychiatry 2008;192:344-50.

Correspondence to: Dr Anick Berard, CHU Sainte-Justine, Research Center, 3175 chenin de la Cote-Ste-Catherine, Montreal, Quebec H3T 1C5, Canada; anick.berard@montreal.ca

Source of funding: Fonds de la Recherche en Sante du Quebec (grant 6263), Reseau Quebecois de Recherche sur I'Usage des Medicaments and the FRSQ Network for the Wellbeing of Children. dominance of postnatal psychiatric disorders in the psychiatric research literature, disorders such as depression are also common during pregnancy, are associated with considerable morbidity for the pregnant woman and can lead to adverse outcomes for the fetus and subsequent poorer long term outcomes for the child. ${ }^{1}$ Although clinicians do use antidepressants during pregnancy, concerns remain about the potential harm of antidepressants for the exposed fetus, including risk of congenital malformations, neonatal syndrome and longer term adverse behavioural outcomes. This paper seeks to investigate whether duration of exposure during the first trimester increases the risk of major congenital malformations using administrative databases and finds no association between antidepressant use during the first trimester and major congenital malformations.
Limitations of this study include the lack of knowledge on whether prescribed antidepressants have been taken (or when they were taken). In addition, the study population is unusual in that nearly half are on welfare benefits. It is unclear why this group had a particularly high rate (8\%) of major congenital malformations but the high rates of other prescribed potentially teratogenic medications (including anticonvulsants and anxiolytics) may have contributed to this finding. There was also a failure to adequately adjust for severity of disorder during pregnancy, limited knowledge of the exact timing of gestational exposure (exposure during different weeks within the first gestation may have a differential effect) and too small a study population to investigate individual antidepressants and their specific risk of congenital malformations. Nevertheless, this study provides some further reassurance to clinicians when prescribing antidepressants during the first trimester and adds to the rapidly changing evidence base in this field. It is difficult but important for clinicians to be up to date with this evidence in order to provide pregnant women with comprehensive information on the risks and benefits of taking medication. Such decisions also continue to be dependent on the psychiatric history and the severity of the antenatal depression.

\section{Dr Louise Howard, PhD, MRCPsych}

Section of Community Mental Health, Health Service and Population Research Department, Institute of Psychiatry, King's College London, London, UK

\section{Competing interests: None.}

1. NICE. Antenatal and postnatal mental health. The NICE guideline on clinical management and service guidance. CG45. London: National Institute for Health and Clinical Excellence, 2007. 